\title{
Comparative Analysis of Quasi-Conformal Deformations in Shape Space
}

\author{
Vahid Taimouri ${ }^{1}$, Huiguang $\mathrm{He}^{2}$, and Jing Hua ${ }^{1}$ \\ ${ }^{1}$ Computer Science, Wayne State University, Detroit, MI 48202, USA \\ ${ }^{2}$ Institute of Automation, CAS, Beijing, 100190, China \\ jinghua@wayne.edu
}

\begin{abstract}
A novel approach based on the shape space concept is proposed to classify quasi-conformal deformations of 3D models. A new metric on the quotient space of meshes is introduced to capture changes of the curvature at each vertex of a simplicial complex during deformation. Then, the deformation curve is obtained by calculating the geodesic curve connecting two shapes in the shape space manifold. In order to compare the deformations, the deformation curves are first transferred to the same part of shape space. And then, the Multi-Dimensional Scaling method is employed to eliminate the redundant dimensions facilitating easy comparison of the deformations. To evaluate our method, some synthetic datasets and 23 datasets of gated images of the left heart ventricle during one heartbeat have been examined. Our experiments show that the algorithm can effectively classify normal and abnormal left heart ventricle deformations in shape space.
\end{abstract}

Keywords: Deformation classification, Shape space, Riemannian metric, Quasi-conformal deformation.

\section{Introduction}

Appropriate deformable shape analysis techniques are of utmost importance for time-varying shape comparison and classification. From a geometric point of view, in order to support efficient shape characterization, a higher level of shape abstraction and information reduction is necessary. The traditional geometrybased approaches towards the shape classification are mainly based on the techniques in which global or local geometrical features are extracted from shapes, and then, a metric is constructed based on bending energy or difference in curvature. Finally, shapes are classified by minimizing the energy functional.

The modern geometry introduces shape space, where coordinates of points in this space represent some generalized properties related to various geometrical properties. In the other words, a shape space is established such that each surface group relates to the same point in shape space, where each deformation sequence is shown by a curve. The length of the curve joining two points determines the similarity between two shapes according to the property which the shape space preserves. The desired characteristics to which shape space is invariant can be induced by choosing an appropriate geometric structure.

T. Jiang et al. (Eds.): MICCAI 2010, Part III, LNCS 6363, pp. $489496,2010$.
(C) Springer-Verlag Berlin Heidelberg 2010 
Invariant to the conformal transformations, a conformal structure can be computed based on the period matrix for manifolds with arbitrary topologies [1]. To induce conformal mappings in $2 \mathrm{D}$ and quasi-conformal mappings in $3 \mathrm{D}$, the Green Coordinates [2] are used in the cage-based space deformation estimation. Continuous Ricci flow 3] conformally deforms a Riemannian metric on a smooth surface such that the Gaussian curvature evolves like a heat diffusion process. Eventually, the Gaussian curvature becomes constant and the limiting Riemannian metric is conformal to the original one. In discrete case, the discrete Ricci flow 5] conformably deforms the circle packing metrics 4] with respect to the Gaussian curvatures. In [6], the geodesic lengths of homotopy classes, measured by Hyperbolic Uniformization metric, are used to determine coordinates of each conformal class in the Teichmüller shape space to classify shapes with negative Euler number. The curvature at each point of a manifold changes according to the deformation characteristics during the deformation.

In contrast, our work is based on the geometric structure presented in 7], which computes all smooth groups of diffeomorphisms mapping two objects together. A quasi-conformal metric based on changes of the curvature at each vertex and an algorithm to classify deformations based on the quasi-conformal metric are proposed.

\section{Deformation Analysis in Shape Space}

Shape Space: Let $\mathbf{G}$ be the space of all immersions with the same connectivity. The deformation of the immersion $\mathbf{M}$ constitutes a curve in this space. Therefore, the tangent vector of $X$, which belongs to the tangent plane of $\mathbf{G}$ at point $\mathbf{M}$, assigns a vector $X_{p}$ to each point $p$ on $\mathbf{M}$. Given a smooth deformation of all points on $\mathbf{M}$, the vector field $X(t)$ is $\partial p(t) / \partial t$ for all points $p$. Depending on the deformation characteristics in which we are interested, various shape spaces having different intrinsic geometries may be defined. Our algorithm to classify deformations is as follows,

1. Mesh registration to find corresponding points on different meshes.

2. Determination of deformation paths in shape space.

3. Transfer of deformation paths in shape space, and dimension reduction.

4. Construction of the 3D spatiotemporal space.

\subsection{Quasi-Conformal Deformation Estimation}

In the Riemannian geometry, a metric is defined as the inner product of two vector fields. Suppose two different deformation fields as $X$ and $Y$, the distance between them are measured by $\langle\langle X, Y\rangle\rangle$ in shape space. Some geometric structures have been established to provide the Isometric and Rigid metrics based on the Riemannian metric definition [7. Here, we propose a quasi-conformal metric within this structure. Let $\Sigma$ be a simplicial complex, and a mapping $f: \Sigma \rightarrow \Re^{3}$ embed $\Sigma$ to the Euclidean space, then $\mathbf{M}=(\Sigma, f)$ is a triangular mesh. Also, let $\left\{X_{p}, X_{q}, X_{r}\right\}$ be corresponding deformations of three vertices of the face $\Delta p q r$ on $\mathbf{M}$. 


\subsection{Quasi-Conformal Metric}

Let $\Delta p q r$ and $\Delta \dot{p} q \dot{r}$ be two triangles such that $p(t)=p(t+\Delta t)=p(t)+X_{p}(t)$, $\dot{q}(t)=q(t+\Delta t)=q(t)+X_{q}(t)$ and $\dot{r}(t)=r(t+\Delta t)=r(t)+X_{r}(t)$. In the Euclidean geometry, if the edge lengths of a triangle are multiplied by the same coefficient, the resulting triangle has the same angles as the original one; therefore,

$$
\frac{\|\dot{p}-\dot{q}\|^{2}}{\|p-q\|^{2}}=\frac{\|\dot{p}-\dot{r}\|^{2}}{\|p-r\|^{2}} .
$$

Eq11yields the following relation,

$$
\frac{\|\dot{p}(t)-\dot{q}(t)\|^{2}-\|p(t)-q(t)\|^{2}}{\|p(t)-q(t)\|^{2}}=\frac{\|p(t)-\dot{r}(t)\|^{2}-\|p(t)-r(t)\|^{2}}{\|p(t)-r(t)\|^{2}} .
$$

The numerator shows the changes of the squared edge length within $\Delta t$. If both sides of the equal sign are divided by $\Delta t$, and also $\Delta t \rightarrow 0$; then, the numerator would be equal to the derivative of the squared edge length. By differentiating $\|p(t)-r(t)\|^{2}, \mathrm{Eq} 3$ will be derived,

$$
\frac{\left\langle p(t)-q(t), X_{p}(t)-X_{q}(t)\right\rangle}{\|p(t)-q(t)\|^{2}}=\frac{\left\langle p(t)-r(t), X_{p}(t)-X_{r}(t)\right\rangle}{\|p(t)-r(t)\|^{2}},
$$

from which $T_{p}(X)$ is achieved as follows,

$$
T_{p}(X)=\left\langle p-q, X_{p}-X_{q}\right\rangle \cdot\|p-r\|^{2}-\left\langle p-r, X_{p}-X_{r}\right\rangle \cdot\|p-q\|^{2} .
$$

Likewise, $T_{q}(X)$ and $T_{r}(X)$ are calculated. To preserve angles of each triangle $\Delta p q r$, a similarity transformation requires that all $T_{p}(X), T_{q}(X)$ and $T_{r}(X)$ vanish during deformation. Thus a conformal metric is obtained as follows,

$$
\begin{gathered}
T_{\Delta p q r}(X)=\left|T_{p}(X)\right|+\left|T_{q}(X)\right|+\left|T_{r}(X)\right|, \\
\langle\langle X, Y\rangle\rangle_{M}^{C}=\sum T_{\Delta p q r}(X) \cdot T_{\Delta p q r}(Y) .
\end{gathered}
$$

Although this metric preserves characteristics of conformal deformations, the shape space geometry of these conformal deformations cannot be reconciled to the characteristics of some non-conformal deformations. For instance, while the heart beats; one part of the heart expands while other portions contract, meaning the heart deformation is not conformal in the strict sense. This necessitates a quasi-conformal shape space which better preserves these non-conformal deformation characteristics. Let $\Delta K_{p}$ be the changes of curvature at the vertex $p$ during deformation, thus $T_{p}(X)$ is redefined as follows,

$$
T_{p}(X)=\Delta K_{p} \cdot\left[\left\langle p-q, X_{p}-X_{q}\right\rangle \cdot\|p-r\|^{2}-\left\langle p-r, X_{p}-X_{r}\right\rangle \cdot\|p-q\|^{2}\right],
$$

and by substituting the new $T_{p}(X)$ from $\mathrm{Eq} 7 \mathbf{7}$ in $\mathrm{Eq} 5$ and $\mathrm{Eq}[6$ the final quasiconformal metric is achieved. In this quasi-conformal metric, changes in the curvature at each vertex are considered such that each vertex with lower curvature changes has less effect in the metric. In order to find the geodesic on the shape space manifold, the energy functional $E(\mathbf{M})=\int\langle\langle X, X\rangle\rangle_{M} d t$ of the curve connecting each pair of meshes is minimized. 


\subsection{Deformation Transfer and Dimension Reduction}

Two deformations are comparable, if their deformation curves are transferred to the same part of shape space. A method based on the parallel transport approach is used for the deformation transfer. Let $\mathbf{M}$ and $\mathbf{N}$ be two immersions, and $X$ and $Y$ be their corresponding deformations, respectively. We sample each deformation path at equidistant points $\mathbf{M}_{i}$ and $\mathbf{N}_{i}$, thus the line segments connecting each pair of sequential samples will be $X_{i}=\Delta \mathbf{M}_{i}$ and $Y_{i}=\Delta \mathbf{N}_{i}$, respectively. Assume the geodesic line $\gamma_{i}$ connects each pair of meshes $\mathbf{M}_{i}$ and $\mathbf{N}_{i}$ in the shape space manifold. The idea is to transfer the deformation $X_{i}$ to $Y_{i}$ along the geodesic curve $\gamma_{i}$ connecting two corresponding immersions.

First, the vector $X_{0}$ is transferred along the geodesic line $\gamma_{0}$ using the parallel transport method to get $\dot{Y}_{0}$ and $\mathbf{N}_{1}=\mathbf{N}_{0}+\dot{Y}_{0}$; then, the geodesic line $\gamma_{1}$, connecting $\mathbf{N}_{1}$ and $\mathbf{M}_{1}$ is achieved and $X_{1}$ is transferred along it to get $\dot{Y}_{1}$ and $\mathbf{N}_{2}=\mathbf{N}_{1}+\dot{Y}_{1}$. Likewise, all the segments are transferred so that the final transferred deformation $\dot{Y}$ is achieved [7].

At this point, all deformation paths have been transferred to the same part in shape space. However, high dimensional shape space prohibits an intuitive perception of deformation comparison. As a result, redundant dimensions of shape space should be eliminated such that the projected deformation paths are as similar as possible to the original deformation paths. To accomplish this, a distance matrix demonstrating distance of each pair of meshes is built based on the defined metric. Then, the Multi-Dimensional Scaling (MDS) method is used to reduce dimensions of shape space and project deformation paths onto a $2 \mathrm{D}$ space, called the 2D spatial space.

\subsection{Deformation Classification}

Although deformations take place in time, we do not have any consideration about time in this deformation structure. To address this issue, time axis is added to the 2D spatial space, as the third dimension. We call this new 3D shape space, spatiotemporal space. Finally, the Locally Linear Embedding (LLE) method is used to mapped 3D deformation curves onto a 2D plane such that each point in this $2 \mathrm{D}$ plane corresponds to one deformation curve in the spatiotemporal space. The more similar two deformations, the closer their corresponding points are in the $2 \mathrm{D}$ plane. By adding time axis as the third dimension to the $2 \mathrm{D}$ spatial space rather than to the original shape space, the time dimension will have more influence on the final deformation projection process as a naturally different dimension.

\section{Experiments on Synthetic Datasets}

In order to evaluate performance of our metric, some objects are deformed in accordance with different deformation patterns; then, the deformations are transferred to other objects. The more accurately the non-conformal deformations are followed by the second object without distortion, the better the metric can 


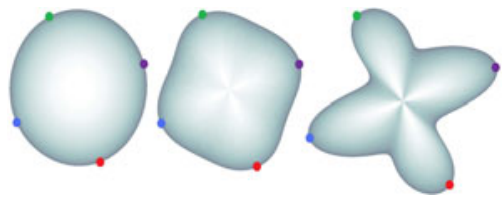

(a)

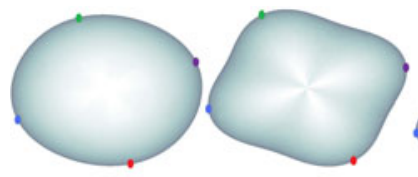

(b)

Fig. 1. a) The non-conformal deformation of a sphere within three time points from left to right. b) The ellipsoid follows the deformation of the sphere.

estimate the intrinsic geometry of shape space and preserve the invariant characteristics of the objects during deformation. Fig 1 a demonstrates a non-conformal deformation on a sphere, which is followed by an ellipsoid (Fig 1, b).

Although the shape deforms non-conformally, the ellipsoid can accurately follow the deformation, and the quasi-conformal metric can circumvent the local minima during optimization of the energy functional by learning the geometry of shape space. We applied the deformation transfer method based on our metric on 60 deformations, all of which were successfully followed by different objects.

In the next step, some different deformations are applied on a torus and classified by the classification algorithm. Since we classify the synthetic datasets, the corresponding points on different meshes are known. Therefore, the mesh registration step can be ignored. Fig 2 illustrates classification of different deformations of a torus, in which only the representative deformations are shown next to solid dots, and the deformations corresponding to the soft dots are not depicted due to the lack of space. The similar deformations will be close together in the $2 \mathrm{D}$ plane.

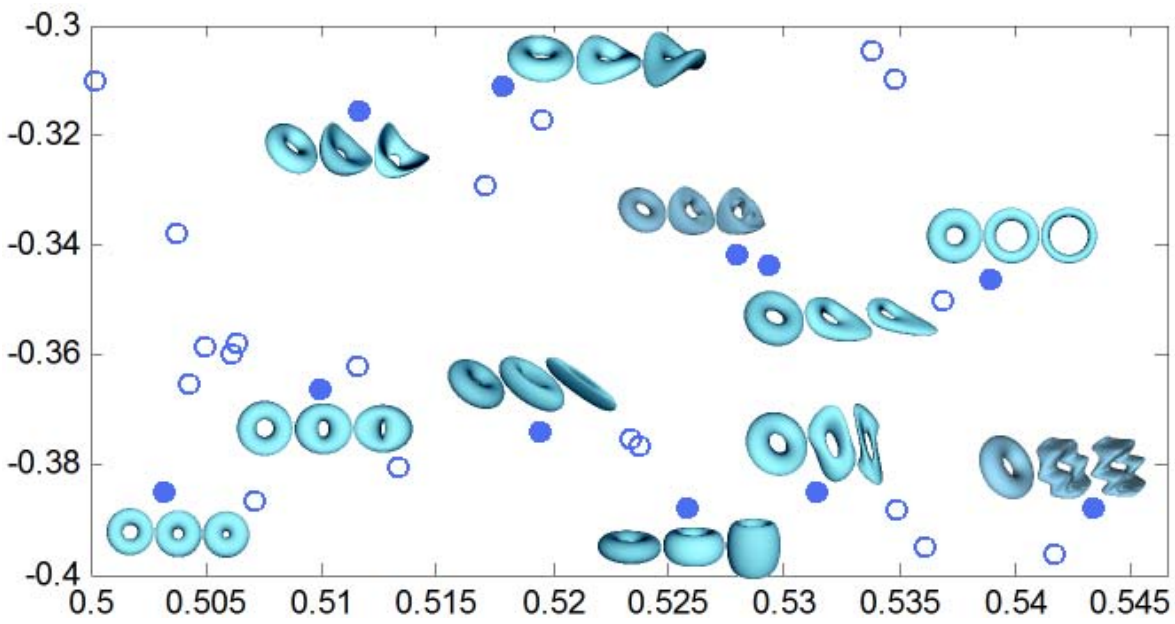

Fig. 2. The classification of different deformations applied on a torus such that each dot corresponds to one deforamtion (Two axes are first and second coordinates of LLE) 
Due to the acquisition procedures, datasets might contain some noise, thus one aspect of classification is about how accurately a classifier can classify noisy datasets. To evaluate this aspect, the points corresponding to different deformations in the $2 \mathrm{D}$ plane are classified into some classes by the $k$-means clustering algorithm. Let $\mathbf{K}$ be the set of all points corresponding to all deformations and $\mathbf{k}_{i}$ be the set of points classified in the group $i^{\text {th }}$ by the $k$-means method. In addition, let $k_{i}$ belong to the set $\mathbf{k}_{i}$, and $l_{i}$ be its assigned label. Eq 8 calculates the statistic $\tau$ quantifying the performance of the algorithm as follows,

$$
\tau=\frac{\sum_{i}\left|\left\{k_{i} \in \mathbf{k}_{i} \mid l_{i}=i\right\}\right|}{|\mathbf{K}|} .
$$

Table. 1 shows the algorithm performance against additive noises with different distributions, which were added to the synthetic genus zero or one meshes with zero, one or two boundaries. As can be seen, the percentage of misclassified deformations is acceptable up to $10 \%$ of additive noise, meaning the pattern of deformations of topologically different meshes can be detected well. Note that, the algorithm shows better performance against the uniform noise compared with the noise with either Gaussian or Poisson distribution.

Table 1. Performance of the algorithm against noisy datasets with different percentages of additive noise variance

\begin{tabular}{llll}
\hline Noise Variance & Uniform Noise & Gaussian Noise & Poisson Noise \\
\hline $1 \%$ & $99.6 \%$ & $99.4 \%$ & $99.2 \%$ \\
$2.5 \%$ & $99.3 \%$ & $97.3 \%$ & $97.2 \%$ \\
$5 \%$ & $96.0 \%$ & $94.4 \%$ & $95.1 \%$ \\
$10 \%$ & $90.1 \%$ & $88.8 \%$ & $89.0 \%$ \\
\hline
\end{tabular}

\section{Application on Heart Motion Analysis}

The proposed algorithm was applied on gated images of the left heart ventricle during one heartbeat. A total of 23 datasets with resolution of $128 \times 128 \times 47 \mathrm{~mm}^{3}$ were acquired from some normal and abnormal subjects. In a preprocessing step, all the datasets are normalized within a bounding box. Subsequently, the intersubject and intra-subject registrations are preformed to find the corresponding points among different meshes. To accomplish this, a total of 16 landmarks are chosen on meshes manually (Fig $\sqrt[3]{3})$, and then the constrained thin plate spline method is used to perform the registration [8,9].

Although the noise distribution of the gated images is approximately Poisson, it might be estimated as Gaussian as well, depending on the number of channels used during the image acquisition. Either of the cases, the proposed method resists against the additive noises well. As can be seen in Fig [3, a, the area connecting the left ventricle to the right one is cut to make a boundary, which is used to set the landmarks. Then, 23 normal and abnormal registered datasets, 9 out 


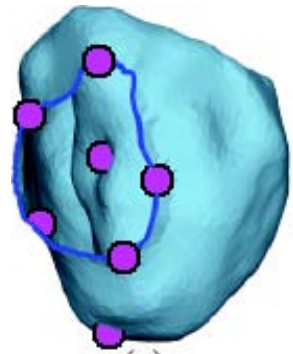

(a)

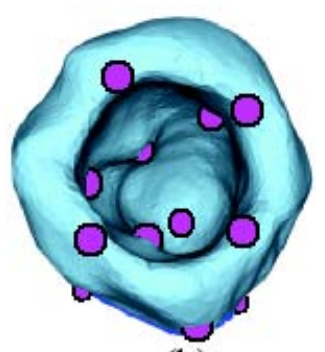

(b)

Fig. 3. The landmarks are chosen, a) on the boundary, b) inside the ventricle

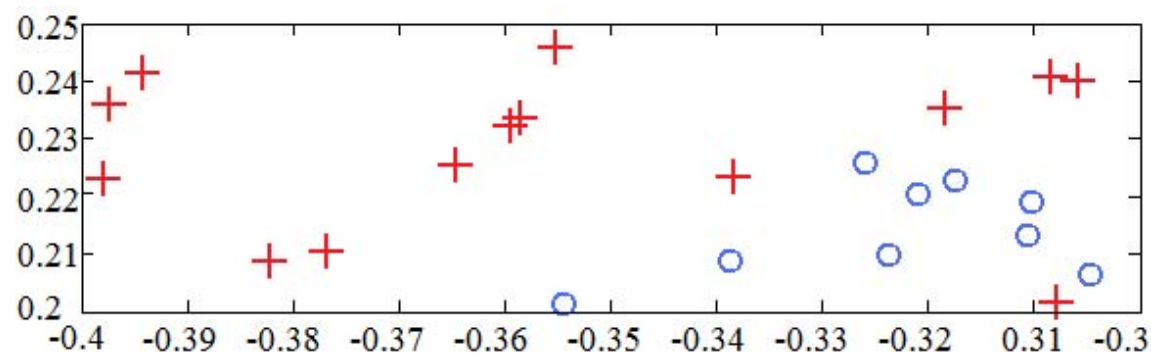

Fig. 4. The classification of normal (circles) and abnormal (crosses) subjects, in which the two axes are the first two coordinates of LLE

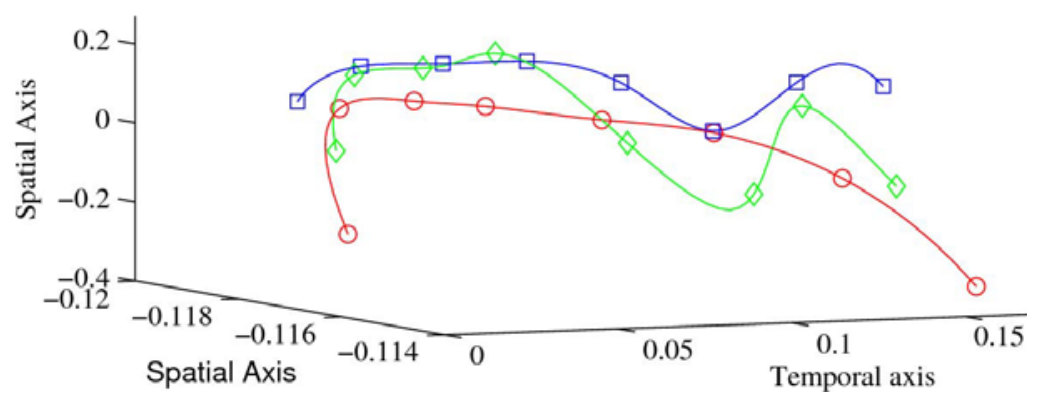

Fig. 5. Two normal subjects (squares and dimonds) and one abnormal subject (circles) in the $3 \mathrm{D}$ spatiotemporal space

of which are normal, are classified by the algorithm. Shown by circles in Fig 4, the normal datasets are almost located in the same part of the 2D plane. Fig [5] demonstrates the normal and abnormal deformations in the $3 \mathrm{D}$ spatiotemporal space, in which the normal deformations follow the similar pattern, which makes their corresponding points in the $2 \mathrm{D}$ plane close to each other. In fact, adding the extra temporal dimension is essential, as it takes into account the speed of the deformation which is of utmost importance in heart diagnosis. 


\section{Conclusion}

In this paper, we have presented an algorithm to effectively classify and compare deformations based on a novel quasi-conformal metric in shape space. To make deformation paths intuitively comparable in shape space, the deformations are transferred and projected into a $2 \mathrm{D}$ plane, where each point corresponds to one deformation. The method has been successfully applied to heart data analysis on differentiating normal and abnormal heart motion.

\section{Acknowledgements}

The authors gratefully acknowledge the support of K. C. Wong Education Foundation, Hong Kong, and the National Science Foundation under the grants NSF IIS-0915933, NSF IIS-0937586 and NSF IIS-0713315.

\section{References}

1. Gu, X., Wang, Y., Yau, S.T.: Computing Conformal Invariants: Period Matrices. Communications in Information and Systems 3, 153-197 (2004)

2. Lipman, Y., Levin, D., Cohen, D.: Green Coordinates. In: ACM SIGGRAPH, vol. 27 (2008)

3. Hamilton, R.S.: Three Manifolds with Positive Ricci Curvature. J. Differential Geometry 17, 255-306 (1982)

4. Collins, C., Stephenson, K.: A Circle Packing Algorithm. Computational Geometry: Theory and Applications 25, 233-256 (2003)

5. Jin, M., Kim, J., Luo, F., Gu, X.: Discrete Surface Ricci Flow. IEEE Trans. on Visualization and Computer Graphics (TVCG) 14, 1030-1043 (2008)

6. Jin, M., Zeng, W., Luo, F., Gu, X.: Computing Teichmüller Shape Space. IEEE Trans. on Visualization and Computer Graphics (TVCG) 15, 504-517 (2009)

7. Kilian, M., Mitra, N.J., Pottmann, H.: Geometric Modeling in Shape Space. In: ACM SIGGRAPH, vol. 26 (2007)

8. Zou, G., Hua, J., Muzik, O.: Non-rigid Surface Registration Using Spherical Thinplate Splines. In: Ayache, N., Ourselin, S., Maeder, A. (eds.) MICCAI 2007, Part I. LNCS, vol. 4791, pp. 367-374. Springer, Heidelberg (2007)

9. Duan, Y., Hua, J., Qin, H.: Interactive Shape Modeling Using Lagrangian Surface Flow. The Visual Compute. 21(5), 279-288 (2005) 